\title{
BMJ Open Reporting quality of randomised controlled trial abstracts among high-impact general medical journals: a review and analysis
}

\author{
Meredith Hays, ${ }^{1,2}$ Mary Andrews, ${ }^{1,2}$ Ramey Wilson, ${ }^{1,2}$ David Callender, ${ }^{2}$ \\ Patrick G O'Malley, ${ }^{1,2}$ Kevin Douglas ${ }^{1,2}$
}

To cite: Hays $\mathrm{M}$, Andrews $\mathrm{M}$, Wilson R, et al. Reporting quality of randomised controlled trial abstracts among high-impact general medical journals: a review and analysis. BMJ Open 2016;6: 011082. doi:10.1136/bmjopen-2016011082

- Prepublication history for this paper is available online. To view these files please visit the journal online (http://dx.doi.org/10.1136/ bmjopen-2016-011082).

Received 8 January 2016 Revised 24 May 2016 Accepted 29 June 2016

CrossMark

\begin{abstract}
${ }^{1}$ Department of Medicine, Uniformed Services University, Bethesda, Maryland, USA

${ }^{2}$ Department of Internal Medicine, Walter Reed National Military Medical Center, Bethesda, Maryland, USA
\end{abstract}

Correspondence to Dr Meredith Hays; mhays24@gmail.com

\section{ABSTRACT}

Objective: The aim of this study was to assess adherence to the Consolidated Standards of Reporting Trials (CONSORT) for Abstracts by five high-impact general medical journals and to assess whether the quality of reporting was homogeneous across these journals.

Design: This is a descriptive, cross-sectional study.

Setting: Randomised controlled trial (RCT) abstracts in five high-impact general medical journals.

Participants: We used up to 100 RCT abstracts published between 2011 and 2014 from each of the following journals: The New England Journal of Medicine (NEJM), the Annals of Internal Medicine (Annals IM), The Lancet, the British Medical Journal (The BMJ) and the Journal of the American Medical Association (JAMA).

Main outcome: The primary outcome was per cent overall adherence to the 19-item CONSORT for Abstracts checklist. Secondary outcomes included per cent adherence in checklist subcategories and assessing homogeneity of reporting quality across the individual journals.

Results: Search results yielded 466 abstracts, 3 of which were later excluded as they were not RCTs. Analysis was performed on 463 abstracts (97 from NEJM, 66 from Annals IM, 100 from The Lancet, 100 from The BMJ, 100 from JAMA). Analysis of all scored items showed an overall adherence of $67 \%$ (95\% Cl 66\% to $68 \%$ ) to the CONSORT for Abstracts checklist. The Lancet had the highest overall adherence rate $(78 \% ; 95 \% \mathrm{Cl} 76 \%$ to $80 \%)$, whereas NEJM had the lowest (55\%; $95 \% \mathrm{Cl} 53 \%$ to $57 \%$ ). Adherence rates to 8 of the checklist items differed by $>25 \%$ between journals.

Conclusions: Among the five highest impact general medical journals, there is variable and incomplete adherence to the CONSORT for Abstracts reporting checklist of randomised trials, with substantial differences between individual journals. Lack of adherence to the CONSORT for Abstracts reporting checklist by high-impact medical journals impedes critical appraisal of important studies. We recommend diligent assessment of adherence to

\section{Strengths and limitations of this study}

- Data were gathered through an objective extraction process.

- Our study benefitted from a large sample size.

- Reviewers were blinded to the journal and randomly assigned articles to score.

- Articles were from 2011 to 2014, providing researchers and editors adequate time to fully implement the checklist guidelines published in 2008.

- Our study conclusions may not be applicable to journals not included in our analysis.

reporting guidelines by authors, reviewers and editors to promote transparency and unbiased reporting of abstracts.

\section{BACKGROUND}

Randomised controlled trials (RCTs) are considered the gold standard for evidence for interventions, but assessing trial validity is dependent on the quality and transparency of the study report. ${ }^{1}{ }^{2}$ This requires the inclusion of key study information in abstracts and manuscripts so that readers can properly assess the validity and generalisability of each study and apply the findings to their patient population. Responsibility extends to peer reviewers and medical journal editors who must verify that the information needed to evaluate study quality is reported in abstracts and manuscripts, and guidelines exist to ensure that the essential elements are reported in the manuscript and the abstract. ${ }^{3-6}$ The abstract may be the only read portion of the study, and without clear reporting, this could lead to misinterpretation and poor patient outcomes. ${ }^{7}$ One study found that as many as $63 \%$ of practising 
internal medicine physicians relied solely on the abstract of general internal medicine or general medical journals. This was consistent for physicians with and without formal epidemiological training. ${ }^{7}$

The Consolidated Standards of Reporting Trials (CONSORT) Statement was initially developed in 1996 to improve reporting of RCTs in journals. ${ }^{3-6} 8$ These guidelines underwent subsequent modification in 2001, 2006 and 2010 to improve the manuscript reporting process. ${ }^{8}$ In 2008, the CONSORT for Abstracts checklist was created as an extension of the original CONSORT Statement in order to improve the reporting of RCT abstracts in journals and conference proceedings thereby allowing readers to quickly assess validity and applicability of a trial. ${ }^{4}$

Editors of high-impact journals have endorsed the use of the CONSORT guidelines to facilitate transparent and unbiased reporting of trial results. ${ }^{9}{ }^{10}$ Articles published in high-impact journals are commonly cited in the medical literature and frequently reported in the lay press; ${ }^{11}$ accordingly, the importance of complete and unbiased reporting in these journals is paramount. However, two previous studies examining high-impact medical journals highlighted the lack of adherence to reporting guidelines in RCT abstracts. ${ }^{12}{ }^{13}$ A number of other studies have also assessed the quality of reporting of abstracts of RCTs using various methodologies, but many of these had factors that limited their overall rigour, such as small sample sizes, limited blinding and a lack of an evaluation of inter-rater agreement. ${ }^{14-21} \mathrm{We}$ conducted this study to rigorously assess the adherence of high-impact, high-visibility general medical journals to the reporting quality standards set forth in the CONSORT for Abstracts checklist. The primary outcome was per cent overall adherence to the 19-item CONSORT for Abstracts checklist. Secondary outcomes included per cent adherence in checklist subcategories and assessing homogeneity of reporting quality across the individual journals.

\section{METHODS}

\section{Search strategy and study selection}

We conducted a descriptive, cross-sectional study of RCT abstracts in five journals with the highest impact factors in $2014 .^{22}$ We included abstracts published between 2011 and 2014 in The New England Journal of Medicine $(N E J M)$, the Annals of Internal Medicine (Annals IM), The Lancet, the British Medical Journal (The BMJ), and the Journal of the American Medical Association (JAMA) that reported the main results of parallel-group RCTs. We excluded observational or cohort studies, interim analyses, economic analyses of RCTs, post-trial follow-up studies, subgroup and secondary analyses of previously reported RCTs, editorials, letters and news reports. An author (KD) not involved in the abstract scoring applied the search strategy (figure 1) on 1 December 2014 to identify up to 100 of the most recent RCTs published in

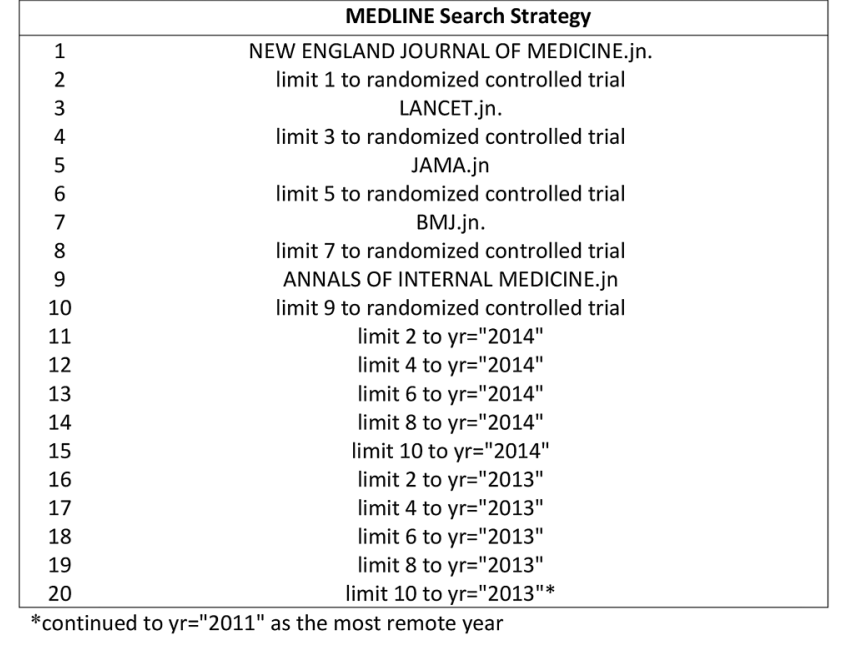

Figure 1 MEDLINE search strategy.

each of the top five general medical journals that met the eligibility criteria. Abstract selection for a particular journal started with those published in 2014, proceeded backwards in time and stopped when 100 abstracts for that journal that met eligibility criteria had been identified or the search for the year 2011 was completed, whichever came first. Abstracts were stored in EndNote X7 (Thomas Reuters, Philadelphia, Pennsylvania, USA). An author (DC) not involved in abstract scoring or data analysis imported abstracts from Endnote X7 into Excel (Microsoft, Redmond, Washington, USA). A customised Excel macro was used to remove PubMed Identification code, journal name, author names and journal-specific subheadings to ensure blinding of reviewers to journal. The same author (DC) maintained the key linking journal number (J1-J5) with journal name. In order to avoid bias, the authors remained blinded to journal identification, through data analysis and synthesis.

A priori sample size calculation using a $\beta$ of 0.2 and an $\alpha$ of 0.05 indicated that a minimum of 58 studies per journal would be needed to detect a difference in adherence between any two journals of $25 \%$ or greater using the two-sample proportions Pearson's $\chi^{2}$ test. The difference of $25 \%$ was chosen by the authors to be the minimum difference they felt would be meaningful between any two journals.

\section{Checklist development, application and inter-rater agreement}

We determined compliance to each aspect of the CONSORT for Abstracts checklist through the use of a 19-item checklist (figure 2). This checklist was developed in an iterative manner by the authors by expanding the published CONSORT for Abstracts checklist to allow for evaluation of each component of the recommendations. ${ }^{4}$ Discrepancies between authors over application of checklist items were resolved by consulting the published explanation of the CONSORT for Abstracts checklist ${ }^{4}$ and by adding instructions and examples to 


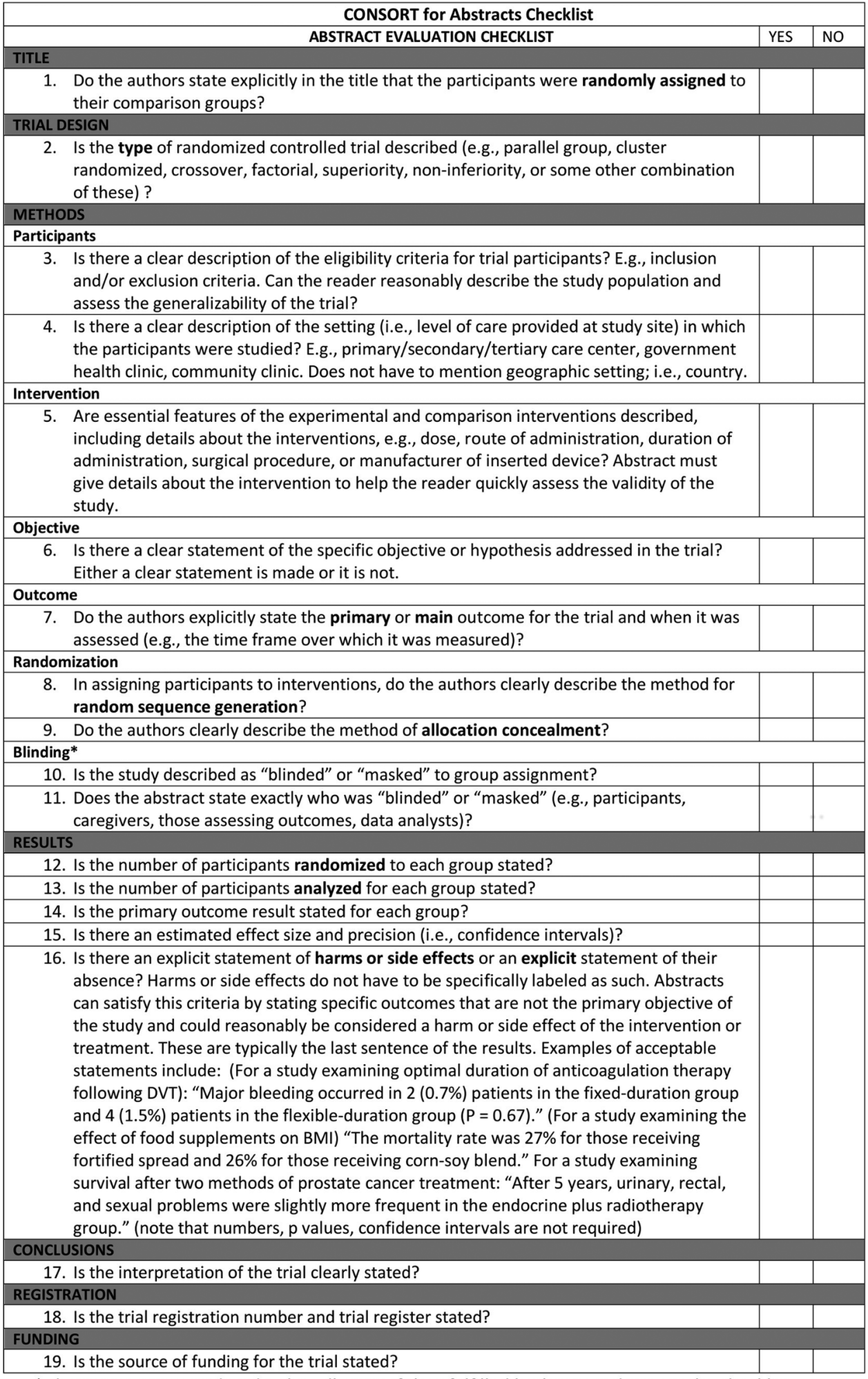

*Abstracts were considered to be adherent if they fulfilled both \#10 and \#11 on the checklist and they were not scored if the answer to \#10 was "no"

Figure 2 CONSORT for Abstracts checklist.

the checklist items as shown in figure 3. Prior to scoring of study abstracts, raw inter-rater agreement for each item on the 19-item checklist was evaluated through a test run of 32 RCT abstracts published prior to 2011 and scored independently by three physician authors (MA,
MH and RW) with graduate-level training in epidemiology and critical appraisal. We chose raw per cent agreement as a measure of inter-rater reliability for simplicity as well as the known difficulties with chancecorrected measures of agreement such as Cohen's $\kappa$, 


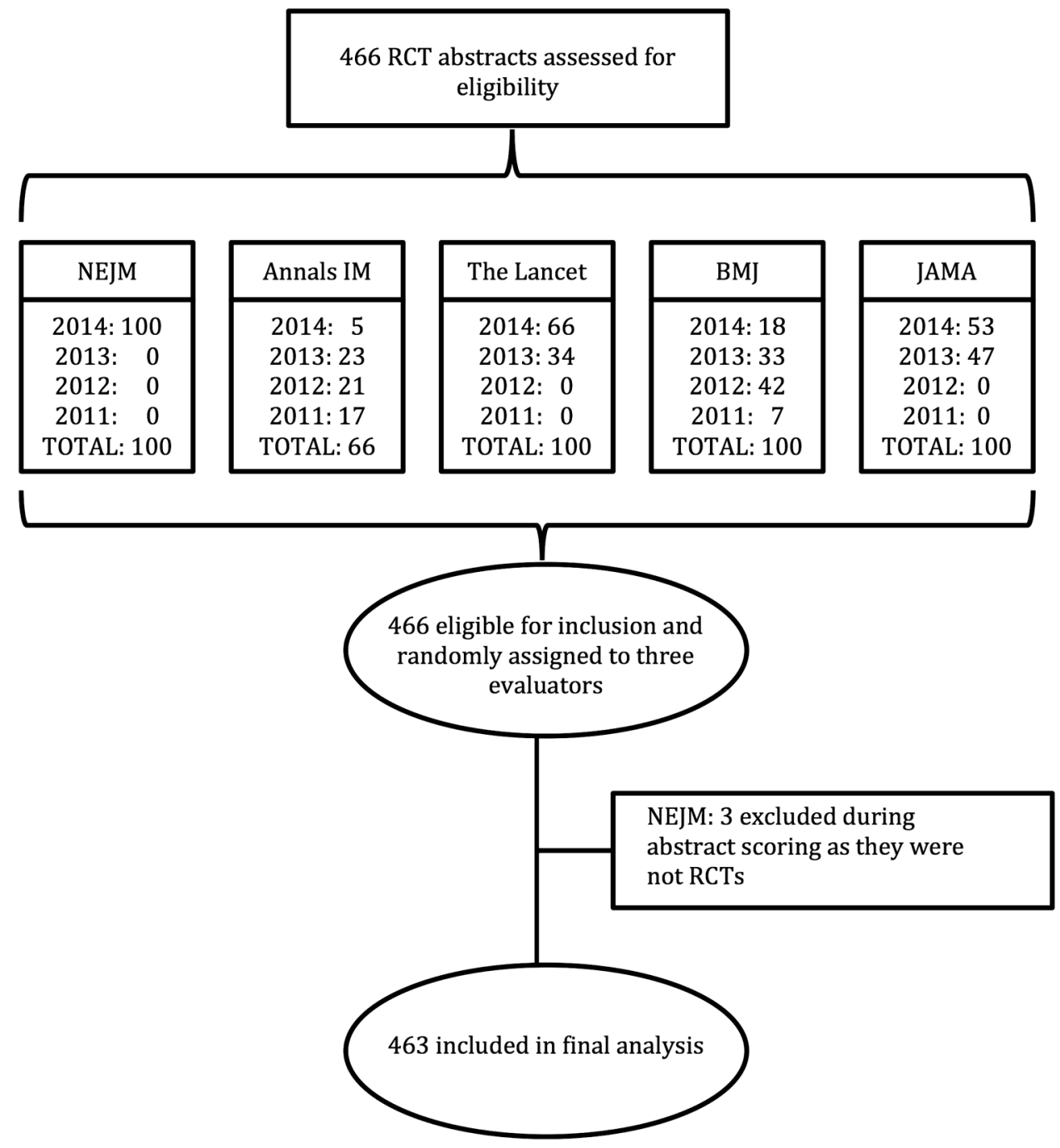

Figure 3 Flow diagram of the study.

which can produce misleadingly low values in the setting of high-per cent agreement. ${ }^{15}{ }^{23-25}$ After ensuring adequate inter-rater agreement with this sample, each study abstract was scored by a single author (MA, MH or RW).

\section{Data extraction and analysis}

Study abstracts were randomly ordered using a computer-generated sequence in Excel (Microsoft) and divided among the three physician authors for review. Each item on the checklist was scored dichotomously (figure 3). The proportion of abstracts adherent to each checklist item was calculated for the entire sample and for the abstracts published in each journal. $\chi^{2}$ test of homogeneity using a significance level of 0.05 was utilized to test the null hypothesis that the proportion of abstracts adherent to checklist items was homogeneous across journals. ${ }^{26}$

Descriptive analysis was performed using STATA, V.13 (StataCorp, College Station, Texas, USA). The proportion of adherence was determined across all journals and checklist items, for all checklist items by individual journal and for all journals by individual checklist item. This represented the average of the adherence rates for all checklist items, weighted by the number of abstracts scored for each item. The number of abstracts scored differed only for the item of blinding, composed of two parts: items 10 and 11. Item 10 looked at generic blinding, whereas item 11 looked for detailed descriptions. If the study was described as blinded or masked to group assignment, the abstract was then rated as adherent if it was stated who was blinded or masked. This was concordant with the CONSORT for Abstracts checklist. If the study was not described as blinded or masked to group assignment, the abstract was not scored for blinding.

The raters (MA, MH and RW) were all trained clinicians with expertise in clinical epidemiology and critical appraisal.

\section{RESULTS}

Journal characteristics

Individual journal characteristics are listed in table 1. 
Table 1 Journal characteristics

\begin{tabular}{llll}
\hline Journal & $\begin{array}{l}\text { Impact } \\
\text { factor* }\end{array}$ & $\begin{array}{l}\text { Use of } \\
\text { CONSORT } \\
\text { endorsed }\end{array}$ & $\begin{array}{l}\text { Overall } \\
\text { adherence† } \\
\text { (\%) }\end{array}$ \\
\hline NEJM & 54.42 & Yes $\ddagger$ & 55 \\
The Annals IM & 16.104 & Yes $\ddagger$ & 70 \\
The Lancet & 39.207 & Yes & 78 \\
The BMJ & 16.378 & Yes & 65 \\
JAMA & 35.289 & Yes $\ddagger$ & 63
\end{tabular}

${ }^{*}$ As determined by ISI Impact Factor 2014.

tOverall adherence to checklist items per journal based on our study findings.

$\ddagger$ No explicit mention of CONSORT for Abstracts in instructions to authors.

NEJM, The New England Journal of Medicine; Annals IM, the Annals of Internal Medicine; The BMJ, the British Medical Journal; JAMA, the Journal of the American Medical Association.

\section{Study characteristics}

PubMed search results yielded 466 study abstracts from the top 5 general medical journals (100 from NEJM, 66 from Annals IM, 100 from The Lancet, 100 from The BMJ and 100 from $J A M A), 3$ of which were later excluded during abstract scoring (NEJM) because they were not RCTs (figure 3). Of note, Annals IM had fewer RCTs $(n=66)$ published during the study timeframe than the other journals in our study. Mean agreement among the 3 reviewers for checklist items was $84 \%$ in the pre-study run-in.

\section{Assessment of reporting quality of the CONSORT for Abstracts checklist items}

Overall, adherence to the CONSORT for Abstract checklist among journals varied (table 2). Analysis of all scored items showed an overall adherence of 67\% (95\% CI $66 \%$ to $68 \%$ ). Adherence was lowest for the reporting of allocation concealment and random sequence generation. Conformity to the checklist was highest for reporting clear interpretations of the trial, stating trial objectives clearly, and including trial registration data. Adherence rates to 8 of the checklist items differed by $>25 \%$ between journals. The Lancet had the highest overall adherence rate $(78 \% ; 95 \%$ CI $76 \%$ to $80 \%)$, whereas $N E J M$ had the lowest $(55 \% ; 95 \%$ CI $53 \%$ to $57 \%)$.

When comparing compliance among the individual journals by checklist items, NEJM lagged behind in more categories (five) than all other journals combined, but led the other journals in reporting of harms. The $B M J$ and JAMA lagged behind the other journals in reporting the funding source of the study. Overall adherence rates displayed substantial heterogeneity among journals. The Lancet had the highest overall adherence rate, whereas NEJM had the lowest (table 3).

\section{DISCUSSION}

This descriptive, cross-sectional analysis examining adherence to the CONSORT for Abstracts checklist in the 5 highest impact general medical journals from 2011 to 2014 showed that the overall adherence was $67 \%$, with markedly lower adherence to individual checklist items (down to $0 \%$ for some items in some journals) and substantial variability across journals. Reporting of allocation concealment and random sequence generation in the abstract text was uncommon $(<25 \%)$ across all journals except The Lancet. The Lancet showed the highest rate of overall adherence and NEJM the lowest ( $78 \%$ and $55 \%$, respectively), though these differences did not meet our prespecified criteria for a meaningful difference of $25 \%$.

Similar to prior work, we found that incomplete adherence to abstract reporting guidelines persists, ${ }^{12} 13$ 27-38 particularly on domains known to influence study results (eg, allocation concealment and blinding). ${ }^{12} \quad 13 \quad 15$ Our study improved on previous studies as a large descriptive, cross-sectional study with a larger sample $(n=463)$ of recently published abstracts in high-impact journals where results receive the highest attention among the clinical research and practising community. This allowed us to show with statistical significance the heterogeneity across the journals. By examining abstracts published between 2011 and 2014, this study provides an updated view of the state of adherence to reporting guidelines since Ghimire et al, ${ }^{12}$ which evaluated abstracts published in 2010. Although comparison with Ghimire's work suggests modest improvement in areas such as blinding, our study showed that adherence is still suboptimal $(<60 \%$ for many items). There is also wide variation in reporting between journals, indicating an opportunity for standardising abstract reporting across the medical literature. Hopewell et $a l^{4}$ examined the impact of editors' implementation of the CONSORT for Abstracts checklist, and their results suggested that effective application of the guidelines led to improved reporting of RCT abstracts. They showed that active implementation of the guidelines led to immediate improvement in the mean number of reported checklist items. The call for improvement in the reporting of RCTs and their abstracts is not new. ${ }^{12} 28-38$ Indeed, many high-impact journals have endorsed the use of the CONSORT Statement and the CONSORT for Abstracts checklist. ${ }^{9}$ Various iterations have been created in an effort to improve reporting across a variety of research venues. $^{36}$

Journals whose editors endorse and enforce the checklist show evidence of improved abstract reporting. ${ }^{13}$ The five high-impact general medical journals examined in our study have endorsed the use of CONSORT as well as the CONSORT for Abstracts checklist, either through their instructions to authors, inclusion on the CONSORT website or both (table 1), although some were more explicit than others. Suboptimal adherence would seem to imply that what is lacking is enforcement. It would be expected that journals of the calibre featured in this study should be better able to enforce guidelines given a presumably more 
Table 2 Adherence by checklist item

\begin{tabular}{llll}
\hline Variable & Observations & Mean & 95\% Cl \\
\hline Title & 463 & 0.79 & 0.75 to 0.83 \\
Trial design & 463 & $0.51^{*}$ & 0.44 to 0.55 \\
Eligibility criteria & 463 & $0.76^{*}$ & 0.72 to 0.80 \\
Study setting & 463 & $0.58^{*}$ & 0.54 to 0.63 \\
Intervention & 463 & 0.87 & 0.83 to 0.90 \\
Objective & 463 & $0.96^{*}$ & 0.93 to 0.97 \\
Primary outcome & 463 & 0.91 & 0.88 to 0.93 \\
Random sequence generation & 463 & 0.19 & 0.15 to 0.22 \\
Allocation concealment & 463 & 0.08 & 0.06 to 0.11 \\
Blinding & 228 & 0.60 & 0.53 to 0.66 \\
Number of randomised per group & 463 & $0.61^{*}$ & 0.56 to 0.65 \\
Number of analysed per group & 463 & $0.44^{*}$ & 0.39 to 0.48 \\
Outcome stated & 463 & 0.83 & 0.79 to 0.86 \\
Effect size/precision & 463 & 0.81 & 0.78 to 0.85 \\
Harms or side effects & 463 & $0.50^{*}$ & 0.45 to 0.54 \\
Interpretation stated & 463 & 0.99 & 0.98 to 1.00 \\
Trial registration & 463 & 0.96 & 0.94 to 0.98 \\
Source of funding & 463 & $0.53^{*}$ & 0.48 to 0.57 \\
Overall adherence & 8099 & 0.67 & 0.66 to 0.68 \\
\hline${ }^{*} \chi^{2}$ test for homogeneity among journals
\end{tabular}

${ }^{*} \chi^{2}$ test for homogeneity among journals was statistically significant $(p<0.001)$.

robust editorial staff and a more rigorous copy editing process. Their failure to enforce the guidelines would suggest that further steps are necessary to maintain adherence. One suggestion would be improved communication of expectations by making the CONSORT for Abstracts checklist a more obvious requirement. Also, our inter-rater agreement on our run-in data was only $84 \%$, which may indicate a need to make the CONSORT checklist less ambiguous for authors, peer reviewers and editors in order to achieve improved adherence.
Our study had several potential limitations. First, our study's conclusions may lack applicability to journals not included in our analysis. Second, our study did not include analysis of temporal trends within our timeframe. If such trends exist, it may be misleading to identify one journal as lagging behind the others if the rates of improvement also differed between journals. Third, we considered all checklist items to be of equal importance, but experts may differ on the relative importance of each item. Finally, our inter-rater agreement averaged only $84 \%$.

Table 3 Adherence to checklist items by individual journal

\begin{tabular}{|c|c|c|c|c|c|}
\hline Variable & NEJM & Annals IM & The Lancet & The BMJ & JAMA \\
\hline Title (\%) & $9^{*}$ & 95 & 97 & 100 & 98 \\
\hline Trial design & $30^{*}$ & 70 & 61 & 59 & 39 \\
\hline Eligibility criteria & 57 & 77 & 83 & 84 & 78 \\
\hline Study setting & $18^{*}$ & 74 & 52 & 85 & 67 \\
\hline Intervention & 78 & 88 & 92 & 88 & 87 \\
\hline Objective & 81 & 100 & 99 & 100 & 99 \\
\hline Primary outcome & 90 & 86 & 95 & 90 & 90 \\
\hline Random sequence generation & 0 & 21 & $62 \dagger$ & 7 & 3 \\
\hline Allocation concealment & 0 & 17 & 22 & 3 & 0 \\
\hline Blinding & $24^{*}$ & 85 & 80 & 73 & $35^{\star}$ \\
\hline Number of randomised per group & $34^{*}$ & 53 & 77 & 66 & 69 \\
\hline Number of analysed per group & 32 & 33 & 65 & 43 & 41 \\
\hline Outcome stated & 85 & 73 & 88 & 74 & 93 \\
\hline Effect size/precision & 77 & 85 & 80 & 81 & 85 \\
\hline Harms or side effects & $69+$ & 38 & 66 & 28 & 43 \\
\hline Interpretation stated & 98 & 100 & 100 & 98 & 100 \\
\hline Trial registration & 97 & 89 & 99 & 95 & 97 \\
\hline Source of funding & 98 & 76 & 94 & $3^{*}$ & $2^{*}$ \\
\hline Overall adherence & 55 & 70 & 78 & 65 & 63 \\
\hline
\end{tabular}

*Journals lagged the majority by $25 \%$ or more $(p<0.001)$ for the category.

†Journals led the majority by $25 \%$ or more $(p<0.001)$ for the category. 
Our study's strengths included robust and reproducible data extraction, blinding reviewers to the journal, randomly assigning articles to reviewers and inclusion of studies from a time period (2011-2014) that provided adequate opportunity for implementation of the CONSORT for Abstracts since its publication in 2008.

In conclusion, the CONSORT for Abstracts is a valuable tool for improving transparency of reporting of clinical trial results. However, our findings indicate a need for systematic editorial and reviewing processes to improve adherence to these guidelines and the transparency of abstract reporting in high-impact medical journals. If we are going to realise the full potential of the CONSORT for Abstracts checklist in improving the quality of abstract reporting, it is critical that editors, peer reviewers and authors commit to its conscientious application.

Contributors KD had full access to all of the data in the study and takes responsibility for the integrity of the data and the accuracy of the data analysis. KD and PGO'M were responsible for the initial study conception, design and protocol. KD, PGO'M, MA, RW, MH and DC made substantial contributions to the initial drafting of the manuscript. KD, PGO'M, MA and $\mathrm{MH}$ made critical revisions of the submitted manuscript. DC was responsible for de-identifying and randomly distributing abstracts to reviewers and maintaining the key to journal identity for each abstract. MA, RW and MH were responsible for abstract scoring. $\mathrm{MH}$ was responsible for the initial draft and overseeing and integrating individual revisions to the manuscript. By-line: $\mathrm{KV}, \mathrm{PGO} M, \mathrm{MA}, \mathrm{RW}, \mathrm{DC}$ and $\mathrm{MH}$.

Funding This research received no specific grant from any funding agency in the public, commercial or not-for-profit sectors.

Disclaimer The views expressed are those of the authors solely and are not to be construed as representing the views of the Department of Defense, the Department of the Navy, the Department of the Army or the Uniformed Services University of the Health Sciences.

\section{Competing interests None declared.}

Ethics approval Patients or patient information was not used in this study. This study was exempt through the Institutional Review Board.

Provenance and peer review Not commissioned; externally peer reviewed.

Data sharing statement Extra data can be accessed via the Dryad data repository at http://datadryad.org/ with the doi:10.5061/dryad.21b04.

Open Access This is an Open Access article distributed in accordance with the Creative Commons Attribution Non Commercial (CC BY-NC 4.0) license, which permits others to distribute, remix, adapt, build upon this work noncommercially, and license their derivative works on different terms, provided the original work is properly cited and the use is non-commercial. See: http:// creativecommons.org/licenses/by-nc/4.0/

\section{REFERENCES}

1. Altman DG. Transparent reporting of trials is essential. Am J Gastroenterol 2013;108:1231-5.

2. Begg $\mathrm{C}$, Cho M, Eastwood $\mathrm{S}$, et al. Improving the quality of reporting of randomized controlled trials. The CONSORT statement. JAMA 1996;276:637-9.

3. Rennie D. How to report randomized controlled trials. The CONSORT statement. JAMA 1996;276:649.

4. Hopewell S, Clarke M, Moher D, et al., CONSORT Group. CONSORT for reporting randomised trials in journal and conference abstracts: explanation and elaboration. PLoS Med 2008;5:e20.

5. Moher D, Schulz KF, Altman DG. The CONSORT statement: revised recommendations for improving the quality of reports of parallel-group randomized trials. Ann Intern Med 2001;134:657-62.
6. Moher D, Hopewell S, Schulz KF, et al. CONSORT 2010 explanation and elaboration: updated guidelines for reporting parallel group randomised trials. BMJ 2010;340:c869.

7. Saint S, Christakis DA, Saha S, et al. Journal reading habits of internists. J Gen Intern Med 2000;15:881-4.

8. History. Consort. http://www.consort-statement.org/about-consort/ history (accessed 26 Mar 2016).

9. CONSORT 2010. Consort. http://www.consort-statement.org/ consort-2010 (accessed 26 Mar 2016)

10. Schulz KF, Altman DG, Moher D. CONSORT 2010 statement: updated guidelines for reporting parallel group randomized trials. BMJ 2010;340:c332.

11. Burns RB, Moskowitz MA, Osband MA, et al. Newspaper reporting of the medical literature. J Gen Intern Med 1995;10:19-24.

12. Ghimire S, Kyung E, Kang W, et al. Assessment of adherence to the CONSORT statement for quality of reports on randomized controlled trial abstracts from four high-impact general medical journals. Trials 2012;13:77.

13. Hopewell S, Ravaud P, Baron G, et al. Effect of editors implementation of CONSORT guidelines on the reporting of abstracts in high impact medical journals: interrupted time series analysis. BMJ 2012;344:e4178.

14. Xu L, Li J, Zhang M, et al. Chinese authors do need CONSORT: reporting quality assessment for five leading Chinese medical journals. Trials 2008;11:75.

15. Nelson H. Systematic reviews to answer health care questions. Philadelphia, PA: Lippincott, Williams \& Wilkins, 2014.

16. Armstrong $\mathrm{R}$, Waters $\mathrm{E}$, Moore $\mathrm{L}$, et al. Improving the reporting of public health intervention research: advancing TREND and CONSORT. J Public Health. 2008;30:103-9.

17. Cui $Q$, Tian J, Song X, et al. Does the CONSORT checklist for abstracts improve the quality of reports of randomized controlled trials on clinical pathways? J Eval Clin Pract 2014;20:827-33

18. Piggot M, McGee H, Feuer D. Has CONSORT improved the reporting of randomized controlled trials in the palliative care literature? A systemic review. Palliat Med 2004;18:32-8.

19. Hopewell S, Eisinga A, Clarke M. Better reporting of randomized trials in biomedical journal and conference abstracts. J Info Sci 2008;34:162-73.

20. Chen $\mathrm{Y}, \mathrm{Li} \mathrm{J}, \mathrm{Ai} \mathrm{C}$, et al. Assessment of the quality of reporting in abstracts of randomised controlled trials published in the five leading Chinese medical journals. PLoS One 2010;5: e11926.

21. Yoon U, Knoblock K. Quality of reporting in sports injury prevention abstracts according to the CONSORT and STROBE criteria in 2005 and 2008. Br J Sports Med 2012;46:202-6.

22. Science Citation Index-Medicine, General \& Internal. Intellectual Property \& Science, Thomson Reuters. http://science. thomsonreuters.com/cgi-bin/jrnlst/jlresults.cgi?PC=D\&SC=PY (accessed 12 Apr 2016).

23. Feinstein AR, Cicchetti DV. High agreement but low kappa: I. The problems of two paradoxes. J Clin Epidemiol 1990;43:543-9.

24. Viera AJ, Garrett JM. Understanding interobserver agreement: the kappa statistic. Fam Med 2005;37:360-3.

25. Gwet K. Kappa statistic is not satisfactory for assessing the extent of agreement between raters. Gaithersburg: STATAxis Consulting, 2002:1-5. http://advancedanalyticsllc.com/irrhbk/ research_papers/kappa_statistic_is_not_satisfactory.pdf (accessed 29 Mar 2016)

26. Hulley SB, Cummings SR, Browner WS, et al. Designing clinical research. 2nd edn. Philadelphia: Lippincott Williams and Wilkins, 2001.

27. Pildal J, Hrobjartsson A, Jorgensen $\mathrm{K}$, et al. Impact of allocation concealment on conclusions drawn from meta-analyses of randomized trials. Int J Epidemiol 2007;36:847-57.

28. Mbuagbaw L, Thabane M, Vanniyasingam T, et al. Improvement in the quality of abstracts in major clinical journals since CONSORT extension for abstracts: a systematic review. Contemp Clin Trials 2014;38:245-50.

29. Dechartres A, Charles P, Hopewell S, et al. Reviews assessing the quality or the reporting of randomized controlled trials are increasing over time but raised questions about how quality is assessed. J Clin Epidemiol 2011;64:136-44.

30. Duff JM, Leather H, Walden EO, et al. Adequacy of published oncology randomized controlled trials to provide therapeutic details needed for clinical application. J Natl Cancer Inst 2010;102:702-5.

31. Dwan K, Altman DG, Arnaiz JA, et al. Systematic review of the empirical evidence of study publication bias and outcome reporting bias. PLoS One 2008;3:e3081. 
32. Glasziou $\mathrm{P}$, Meats $\mathrm{E}$, Heneghan $\mathrm{C}$, et al. What is missing from descriptions of treatment in trials and reviews? BMJ 2008;336:1472-4.

33. Ioannidis JP. Adverse events in randomized trials: neglected, restricted, distorted, and silenced. Arch Intern Med 2009;169:1737-9.

34. Mathieu S, Boutron I, Moher D, et al. Comparison of registered and published primary outcomes in randomized controlled trials. JAMA 2009;302:977-84.

35. You B, Gan HK, Pond G, et al. Consistency in the analysis and reporting of primary end points in oncology randomized controlled trials from registration to publication: a systematic review. J Clin Oncol 2012;30:210-16.
36. Ghimire S, Kyung $\mathrm{E}$, Lee $\mathrm{H}$, et al. Oncology trial abstracts showed suboptimal improvement in reporting: a comparative before-and-after evaluation using CONSORT for Abstract guidelines. J Clin Epidemiol 2014;67:658-66.

37. Berwanger $O$, Ribeiro RA, Finkelsztejn $A$, et al. The quality of reporting trial abstracts is suboptimal: survey of major general medical journals. J Clin Epidemiol 2009;62: 387-92.

38. Des Jarlais DC, Lyles C, Crepaz N. Improving the reporting of quality of nonrandomized evaluations of behavioral and public health interventions: the TREND statement. Am J Public Health 2004;94:361-6. 